Research Article

\title{
Adaptive Backstepping Sliding Mode Control of the Hybrid Conveying Mechanism with Mismatched Disturbances via Nonlinear Disturbance Observers
}

\author{
Wei Yuan, ${ }^{1,2}$ Guoqin Gao ${ }^{1 D},{ }^{1}$ and Jianzhen $\mathrm{Li}^{2}$ \\ ${ }^{1}$ School of Electrical and Information Engineering, Jiangsu University, Zhenjiang 212013, China \\ ${ }^{2}$ School of Electronic Information, Jiangsu University of Science and Technology, Zhenjiang 212003, China \\ Correspondence should be addressed to Guoqin Gao; gqgao@ujs.edu.cn
}

Received 18 December 2019; Accepted 30 June 2020; Published 25 August 2020

Academic Editor: Ruben Puche-Panadero

Copyright (c) 2020 Wei Yuan et al. This is an open access article distributed under the Creative Commons Attribution License, which permits unrestricted use, distribution, and reproduction in any medium, provided the original work is properly cited.

An adaptive backstepping sliding mode controller combined with a nonlinear disturbance observer is designed for trajectory tracking of the electrically driven hybrid conveying mechanism with mismatched disturbances. A nonlinear disturbance observer is constructed for estimation and compensation of the mismatched and matched disturbances. Then, a hybrid control scheme is designed by combining the adaptive backstepping sliding mode controller and the mentioned observer. The Lyapunov candidate functions are utilized to derive the control and adaptive law. According to the simulation and experimental results, superior tracking performance could be obtained through the presented control scheme compared with conventional backstepping sliding mode control. Meanwhile, the presented control scheme can effectively reduce the chattering problem and improve tracking precision.

\section{Introduction}

The performance of the hybrid electrocoating conveying mechanism as the fundamental equipment of the coating process determines the quality and production efficiency of the electrocoating [1]. The hybrid conveying mechanism (HCM) not only removes the roof airbag completely but also has the advantages of wide applicability, flexibility, and strong loading capacity, etc. The HCM as a combination of serial and parallel mechanisms contains the advantages of both of them. The HCM not only improves the poor flexibility, cramped workspace, and other disadvantages of the parallel mechanism but also compensates for the shortcomings of the serial mechanism including low load capacity and slow response. Although the HCM system could be considered as an important development branch of mechanical research, it is a highly nonlinear, strongly coupled, time varying system, and parametric uncertainties. Meanwhile, due to external disturbances such as the fluid resistance, joint friction, variable load, and motor torque disturbance in practical applications, the high-performance trajectory tracking control could not be easily achieved [2].

Many control strategies have been proposed to reject disturbances for parallel and hybrid mechanism, such as proportional integral derivative (PID) control [3], computed torque control [4], fuzzy control [5], robust control [6], backstepping control [7], and sliding mode control (SMC) $[8,9]$. The abovementioned researches are mainly to deal with matched disturbances. However, the external disturbances and the motor control voltage are not in the same channel for the electrically driven hybrid conveying mechanism. Typical "mismatched" disturbances occur in this mechanism that causes great challenges for designing a high-performance tracking controller. For the mismatched disturbances, many control methods have been developed, such as robust control [10], LQR control [11], sliding mode control $[12,13]$, integral sliding mode control $[14,15]$, and backstepping control [16].

Backstepping as an effective control strategy for rejecting matched and mismatched disturbances is a step-by-step 
recursive design method. However, the robustness could not be attained through the conventional backstepping control method. Recently, SMC has been widely used due to its fast response, easy implementation, insensitivity to parametric uncertainty, and disturbances [8]. Thus, backstepping and SMC methods have been combined to increase the system robustness against matched and mismatched disturbances. This approach has been extensively employed in various robot control systems [17-21]. Ma et al. have proposed a control law combining SMC and backstepping method for flexible-joint manipulator with mismatched disturbances [19]. Chen et al. have combined SMC with backstepping to increase the system robustness against disturbances and uncertainty in the trajectory tracking issue of wheeled mobile robots [20]. In [21], an adaptive backstepping SMC scheme has been constructed for the electrohydraulic elastic manipulator with mismatched uncertainties, which improves the rigidity and antidisturbance capability of the system. However, the chattering phenomenon is the main drawback of the SMC, which has not been well studied in the abovementioned works.

In recent years, nonlinear disturbance observer (NDO) has received more and more attention [22-24]. Some researchers have combined NDO with SMC to construct a hybrid control strategy named NDO based SMC. It can effectively reject disturbance and alleviate the chattering problem. This approach has been extensively employed in practical systems like pedestrian robots, underwater vehicles, and ankle rehabilitation robots [25-27]. In this approach, NDO has been utilized for the estimation of the matched disturbance. Then, the SMC law is designed based on this estimation. After the feedforward compensation, only a comparatively small switching gain is required. This NDO based control schemes can also be extended to deal with mismatched disturbances [28-30]. In [31], an NDO based backstepping SMC strategy has been proposed for tracking control knee exoskeleton, but this work is limited to dealing with matching disturbances. To the best of our knowledge, the trajectory tracking problem for the electrically driven hybrid conveying mechanism with mismatched disturbances has not been fully studied.

In this study, a novel hybrid control scheme consisting of backstepping, SMC, and NDO is constructed for the trajectory tracking problem of HCM with matched and mismatched disturbances. In the design procedure, firstly, NDO is utilized to estimate matched and mismatched disturbances. Secondly, the backstepping method is used to design control laws. Then, in order to improve the robustness of the system, the mismatched disturbance estimation is introduced into the virtual control laws to compensate for the mismatched disturbance. Finally, the sliding mode control is introduced in the last step, and an adaptive law is designed to estimate the switching gain of the sliding mode control. The Lyapunov candidate functions of every step are designed to ensure the asymptotical stability of the whole system. The proposed an adaptive backstepping sliding mode control based on nonlinear disturbance observer (ABSMC + NDO) has two main advantages: First, the NDO is utilized to compensate for the mismatched disturbances in the virtual control law. Second, it not only alleviates the chattering problem but also improves tracking precision.

The organization of this paper is illustrated as follows. The dynamic model of the hybrid conveying mechanism is introduced in Section2. Section 3 presents a disturbance observer based adaptive backstepping SMC scheme. The simulation results are presented in Section 4. Section 5 presents the experimental results. Finally, the paper conclusions are outlined in Section 6.

\section{Dynamic Model}

The hybrid electrocoating conveying mechanism is shown in Figure 1. It includes two relatively independent running and lifting-turnover mechanisms. In this mechanism, two sets of symmetric parallel lifting-turnover mechanisms are connected through a connecting rod. A vehicle body-fixing frame is placed on the connecting rod, and the white body to be painted is placed on the fixing frame. The lifting-turnover mechanism as the main body of the hybrid automobile electrocoating conveying mechanism has a significant influence on electrophoretic coating quality and high control demands. Thus, it is considered as the research object. The servo motors are employed to drive the hybrid conveying mechanism. The lifting motor is connected to the ball screw to move the body-fixing frame up and down. The active wheel motor is connected to the speed reducer to turn the body-fixing frame. Thereby, the white body on the bodyfixing frame moves in accordance with the desired trajectory.

The Lagrangian-Euler method is employed to extract the dynamic of the HCM. The obtained dynamic model could be described as follows [2]:

$$
\widehat{\mathbf{M}}(\mathbf{q}) \ddot{q}+\widehat{\mathbf{C}}(\mathbf{q}, \dot{q}) \dot{q}+\widehat{\mathbf{G}}(\mathbf{q})+\mathbf{w}_{1}=\boldsymbol{\tau}+\mathbf{d}_{1 \mathrm{ex}},
$$

where $\mathbf{q}=\left(l_{1}, l_{2}, l_{3}, l_{4}, \phi_{1}, \phi_{2}\right)^{T}$ is the joint space position vector. The inertial matrix, Coriolis and centrifugal force, and gravity vector are denoted by $\widehat{\mathbf{M}}(\mathbf{q}), \widehat{\mathbf{C}}(\mathbf{q}, \dot{q})$ and $\widehat{\mathbf{G}}(\mathbf{q})$, respectively. $\mathbf{w}_{1}$ denotes the model uncertainties of the HCM including parameter perturbation and unmodeled dynamics. $\mathbf{d}_{1 \mathrm{ex}}$ denotes the external disturbance vector of the mechanism, such as the fluid resistance, joint friction, and load. $\tau$ denotes the joint torque vector of the actuator.

The model of the motor could be written as follows [5]:

$$
\widehat{\mathbf{L}} \frac{\mathrm{d} \mathbf{i}}{\mathrm{d} t}+\widehat{\mathbf{R}} \cdot \mathbf{i}+\widehat{\mathbf{K}}_{\mathbf{b}} \dot{q}+\mathbf{w}_{2}=\mathbf{u}+\mathbf{d}_{2 \mathrm{ex}}
$$

where $\mathbf{i}$ is the armature current of the motor, $\widehat{\mathbf{L}}$ is the electrical inductance value of the motor, $\widehat{\mathbf{R}}$ denotes the equivalent electrical resistance of the motor, $\widehat{\mathbf{K}}_{b}$ is the back electromotive force factor of the motor, $\mathbf{w}_{2}$ denotes the model uncertainties of the motor, $\mathbf{u}$ is the control voltage vector, and $\mathbf{d}_{2 \mathrm{ex}}$ is the voltage disturbance vector.

According to the physical characteristics of the motor, the torque vector $\tau$ is given as follows:

$$
\tau=\mathbf{n} \widehat{\mathbf{K}}_{\mathbf{t}} \mathbf{i}
$$

where $\widehat{\mathbf{K}}_{t}$ is the constant torque coefficient vector of each joint motor, $\mathbf{n}$ is the ratio vector of each joint. 


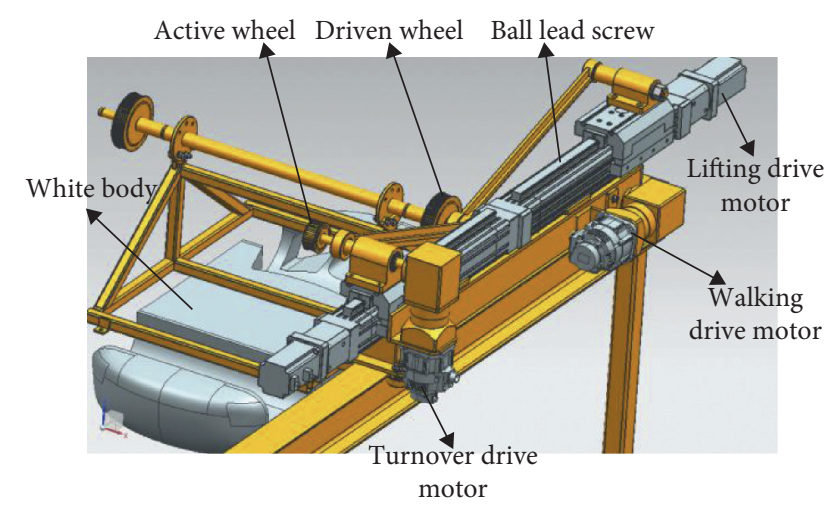

FIGURE 1: The mechanism schematics of HCM.

By combining the uncertainties and disturbances, the following lumped disturbances are obtained:

$$
\begin{aligned}
& \mathbf{d}_{1}=\widehat{\mathbf{K}}_{\mathbf{t}}^{-1}\left(\mathbf{d}_{1 \mathrm{ex}}-\mathbf{w}_{1}\right), \\
& \mathbf{d}_{2}=\widehat{\mathbf{L}}^{-1}\left(\mathbf{d}_{2 \mathrm{ex}}-\mathbf{w}_{2}\right),
\end{aligned}
$$

where $\mathbf{d}_{1}$ and $\mathbf{d}_{2}$ denote the lumped disturbances of the HCM and the motor, respectively.

Considering the dynamic model of HCM given in (1), the motor model given in (2) and the lumped disturbances (4), the dynamic of the electrically driven HCM could be obtained as follows:

$$
\left\{\begin{array}{l}
\mathbf{M}(\mathbf{q}) \ddot{q}+\mathbf{C}(\mathbf{q}, \dot{q}) \dot{q}+\mathbf{G}(\mathbf{q})=\mathbf{i}+\mathbf{d}_{1}, \\
\frac{\mathrm{d} \mathbf{i}}{\mathrm{d} t}+\mathbf{R} \cdot \mathbf{i}+\mathbf{K}_{E} \dot{q}=\hat{\mathbf{L}}^{-1} \mathbf{u}+\mathbf{d}_{2},
\end{array}\right.
$$

where $\mathbf{M}(\mathbf{q})=\left(\mathbf{n} \widehat{\mathbf{K}}_{t}\right)^{-1} \widehat{\mathbf{M}}(\mathbf{q}), \mathbf{C}(\mathbf{q}, \dot{q})=\left(\mathbf{n} \widehat{\mathbf{K}}_{t}\right)^{-1} \widehat{\mathbf{C}}(\mathbf{q}, \dot{q}), \mathbf{G}$ $(\mathbf{q})=\left(\mathbf{n} \widehat{\mathbf{K}}_{t}\right)^{-1} \widehat{\mathbf{G}}(\mathbf{q})$, and $\mathbf{R}=\widehat{\mathbf{L}}^{-1} \widehat{\mathbf{R}}, \mathbf{K}_{E}=\widehat{\mathbf{L}}^{-1} \widehat{\mathbf{K}}_{E}$.

The dynamic properties of the HCM are given in the following: $\dot{M}(\mathbf{q})-2 \mathbf{C}(\mathbf{q}, \dot{q})$ is a skew symmetric matrix, and $\mathbf{M}(\mathbf{q})$ is a symmetric positive definite matrix [24].

Consider that the state variables are defined as follows:

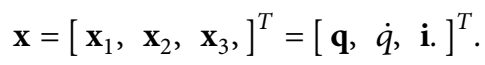

Now, the dynamics (5) could be described with the following state space equations:

$$
\left\{\begin{array}{l}
\dot{x}_{1}=\mathbf{x}_{2}, \\
\dot{x}_{2}=\mathbf{M}(\mathbf{q})^{-1}\left(-\mathbf{C}(\mathbf{q}, \dot{q}) \mathbf{x}_{2}-\mathbf{G}(\mathbf{q})+\mathbf{x}_{3}+\mathbf{d}_{1}\right), \\
\dot{x}_{3}=-\mathbf{R x}_{3}-\mathbf{K}_{E} \mathbf{x}_{2}+\hat{\mathbf{L}}^{-1} \mathbf{u}+\mathbf{d}_{2} .
\end{array}\right.
$$

It could be concluded from equation (7) that the system control input $\mathbf{u}$ and the mismatched disturbances $\mathbf{d}_{1}$ are not on the same channel. Thus, disturbances could not be directly compensated from the control input channel. This could be considered as a typical mismatched disturbances problem.

\section{Control Strategy}

In this section, an NDO-based adaptive backstepping SMC is developed for the HCM (7). The schematic diagram of the presented ABSMC + NDO scheme for the HCM is shown in Figure 2.

3.1. Nonlinear Disturbance Observer Design. Consider that the dynamics (7) is described with the following matrix form:

where

$$
\dot{x}=f(\mathbf{x})+g_{1}(\mathbf{x}) \mathbf{u}+g_{2}(\mathbf{x}) \mathbf{d},
$$

$$
f(\mathbf{x})=\left[\begin{array}{c}
\mathbf{x}_{2}, \\
\mathbf{M}(\mathbf{q})^{-1}\left(-\mathbf{C}(\mathbf{q}, \dot{q}) \mathbf{x}_{2}-\mathbf{G}(\mathbf{q})+\mathbf{x}_{3}\right), \\
-\mathbf{R} \mathbf{x}_{3}-\mathbf{K}_{b} \mathbf{x}_{2},
\end{array}\right],
$$

$g_{1}(\mathbf{x})=\left[\begin{array}{c}0 \\ 0 \\ \hat{L}^{-1}\end{array}\right], g_{2}(\mathbf{x})=\left[\begin{array}{lll}1 & 0 & 0 \\ 0 & 1 & 0 \\ 0 & 0 & 1\end{array}\right], \mathbf{d}=\left[\begin{array}{c}0 \\ \mathbf{d}_{1} \\ \mathbf{d}_{2}\end{array}\right]$.

Assume that the disturbances $\mathbf{d}_{1}$ and $\mathbf{d}_{2}$ are bounded and slowly time varying. For disturbance estimation in the electrically driven HCM (8), the following NDO is utilized $[2,22]$ :

$$
\left\{\begin{array}{l}
\dot{z}=-l(\mathbf{x}) g_{2}(\mathbf{x}) \mathbf{z}-l(\mathbf{x})\left[g_{2}(\mathbf{x}) p(\mathbf{x})+f(\mathbf{x})+g_{1}(\mathbf{x}) \mathbf{u}\right] \\
\widehat{\mathbf{d}}=\mathbf{z}+p(\mathbf{x})
\end{array}\right.
$$

where $\widehat{\mathbf{d}}$ denotes the estimated disturbance, $\mathbf{z}$ denotes the auxiliary vector, and $l(\mathbf{x})$ denotes the observer gain. The term $p(\mathbf{x})$ should be appropriately designed and $l(\mathbf{x})$ is determined by $l(\mathbf{x})=\partial p(\mathbf{x}) / \partial x$.

Consider that the disturbance observer error $\widetilde{\mathbf{d}}$ is defined as $\widetilde{\mathbf{d}}=\mathbf{d}-\widehat{\mathbf{d}}$.

According to equation (9), the error dynamics are as follows:

$$
\begin{aligned}
\dot{\tilde{\mathbf{d}}}= & \dot{d}-\hat{\mathbf{d}} \\
= & -\dot{z}-\frac{\partial p(\mathbf{x})}{\partial x} \dot{x} \\
= & l(\mathbf{x}) g_{2}(\mathbf{x}) \mathbf{z}+l(\mathbf{x})\left[g_{2}(\mathbf{x}) p(\mathbf{x})+f(\mathbf{x})+g_{1}(\mathbf{x}) \mathbf{u}\right] \\
& -l(\mathbf{x})\left[f(\mathbf{x})+g_{1}(\mathbf{x}) \mathbf{u}+g_{2}(\mathbf{x}) \mathbf{d}\right] \\
= & l(\mathbf{x}) g_{2}(\mathbf{x}) \widehat{\mathbf{d}}-l(\mathbf{x}) g_{2}(\mathbf{x}) \mathbf{d} \\
= & -l(\mathbf{x}) g_{2}(\mathbf{x}) \tilde{\mathbf{d}} .
\end{aligned}
$$

According to equation (10), the disturbance estimation error will tend to zero by proper selection of $l(\mathbf{x})$. In this paper, $l(\mathbf{x})$ is chosen as follows:

$$
l(\mathbf{x})=\operatorname{diag}\left(p_{1}, p_{2}, p_{3}\right),
$$

where $p_{1}, p_{2}, p_{3}$ are positive constants.

Consider a Lyapunov function as follows:

$$
V_{0}=\frac{1}{2} \widetilde{\mathbf{d}}^{T} \widetilde{\mathbf{d}} \text {. }
$$




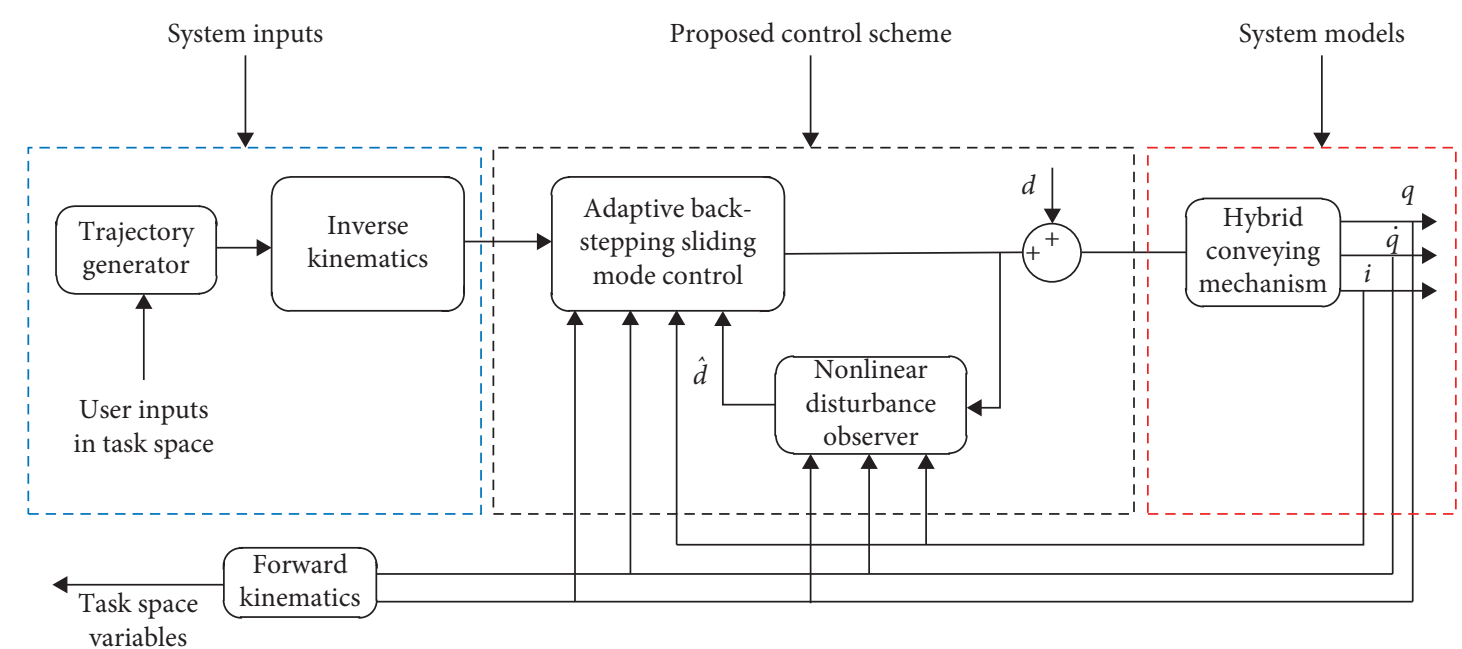

FIgURE 2: Schematic diagram of ABSMC + NDO for the hybrid conveying mechanism.

According to equations (11) and (12), the time derivative of $V_{0}$ could be written as follows:

$$
\dot{V}_{0}=\tilde{\mathbf{d}}^{T} \dot{\tilde{\mathbf{d}}}=-\tilde{\mathbf{d}}_{1}^{T} p_{2} \tilde{\mathbf{d}}_{1}-\tilde{\mathbf{d}}_{2}^{T} p_{3} \tilde{\mathbf{d}}_{2} \leq 0 .
$$

It could be concluded from (13) that the NDO system (10) is asymptotically stable.

3.2. Hybrid Controller Design. In this subsection, the recursive procedure is employed to design a hybrid controller.

Step 1. Define $\mathbf{z}_{1}=\mathbf{x}_{1}-\mathbf{x}_{d}$ as the tracking error, where the desired position is denoted by $\mathbf{x}_{d}$. Now, we have the following:

$$
\dot{z}_{1}=\dot{x}_{1}-\dot{x}_{d}
$$

Define a Lyapunov candidate function as follows:

$$
V_{1}=\frac{1}{2} \mathbf{z}_{1}^{T} \mathbf{z}_{1}
$$

Differentiating from both sides of (15), yields the following:

$$
\dot{V}_{1}=\mathbf{z}_{1}^{T} \dot{z}_{1}=\mathbf{z}_{1}^{T}\left(\dot{x}_{1}-\dot{x}_{d}\right)
$$

Define

$$
\mathbf{z}_{2}=\mathbf{x}_{2}-\mathbf{a}_{1},
$$

where the first virtual control law $\mathbf{a}_{1}$ is defined as follows:

$$
\mathbf{a}_{1}=-\mathbf{c}_{1} \mathbf{z}_{1}+\dot{x}_{d},
$$

where $\mathbf{c}_{1} \in \mathbb{R}^{n \times n}$ is a positive definite diagonal matrix.

Substituting equations (17) and (18) into equation (16), yields the following:

$$
\dot{V}_{1}=\mathbf{z}_{1}^{T} \dot{z}_{1}=\mathbf{z}_{1}^{T}\left(\mathbf{z}_{2}-\mathbf{c}_{1} \mathbf{z}_{1}\right)=-\mathbf{z}_{1}^{T} \mathbf{c}_{1} \mathbf{z}_{1}+\mathbf{z}_{1}^{T} \mathbf{z}_{2} .
$$

If $\mathbf{z}_{2}=0$, then $\dot{V}_{1} \leq 0$. Thus, the control law design should be redesigned.

Step 2. According to equations (7) and (17), the time derivative of $\mathbf{z}_{2}$ is calculated as follows:

$$
\begin{aligned}
\dot{z}_{2} & =\dot{x}_{2}-\dot{a}_{1} \\
& =\dot{x}_{2}-\dot{a}_{1} \\
& =-\mathbf{M}^{-1} \mathbf{C} \mathbf{x}_{2}-\mathbf{M}^{-1} \mathbf{G}+\mathbf{M}^{-1} \mathbf{x}_{3}+\mathbf{M}^{-1} \mathbf{d}_{1}-\dot{a}_{1} .
\end{aligned}
$$

Consider the Lyapunov candidate function as follows:

$$
V_{2}=V_{1}+\frac{1}{2} \mathbf{z}_{2}^{T} \mathbf{M} \mathbf{z}_{2}
$$

Differentiating from both sides of (21), yields the following:

$$
\begin{aligned}
\dot{V}_{2} & =\dot{V}_{1}+\frac{1}{2} \mathbf{z}_{2}^{T} \dot{M} \mathbf{z}_{2}+\mathbf{z}_{2}^{T} \mathbf{M} \dot{z}_{2} \\
& =-\mathbf{z}_{1}^{T} \mathbf{c}_{1} \mathbf{z}_{1}+\mathbf{z}_{1}^{T} \mathbf{z}_{2}+\frac{1}{2} \mathbf{z}_{2}^{T} \dot{M} \mathbf{z}_{2}+\mathbf{z}_{2}^{T} \mathbf{M}\left(-\mathbf{M}^{-1} \mathbf{C} \mathbf{x}_{2}-\mathbf{M}^{-1} \mathbf{G}+\mathbf{M}^{-1} \mathbf{x}_{3}+\mathbf{M}^{-1} \mathbf{d}_{1}-\dot{a}_{1}\right) \\
& =-\mathbf{z}_{1}^{T} \mathbf{c}_{1} \mathbf{z}_{1}+\mathbf{z}_{1}^{T} \mathbf{z}_{2}+\frac{1}{2} \mathbf{z}_{2}^{T} \dot{M} \mathbf{z}_{2}+\mathbf{z}_{2}^{T}\left(-\mathbf{C} \mathbf{z}_{2}-\mathbf{C} \mathbf{a}_{1}-\mathbf{G}+\mathbf{x}_{3}+\mathbf{d}_{1}-\mathbf{M} \dot{a}_{1}\right)
\end{aligned}
$$


According to the property of (5), we have the following: $\dot{V}_{2}=-\mathbf{z}_{1}^{T} \mathbf{c}_{1} \mathbf{z}_{1}+\mathbf{z}_{1}^{T} \mathbf{z}_{2}+\mathbf{z}_{2}^{T}\left(-\mathbf{C} \mathbf{a}_{1}-\mathbf{G}+\mathbf{x}_{3}+\mathbf{d}_{1}-\mathbf{M} \dot{a}_{1}\right)$.

Define

$$
\mathbf{z}_{3}=\mathbf{x}_{3}-\mathbf{a}_{2},
$$

where the virtual control law $\mathbf{a}_{2}$ is designed as follows:

$$
\mathbf{a}_{2}=\mathbf{C} \mathbf{a}_{1}+\mathbf{G}-\widehat{\mathbf{d}}_{1}+\mathbf{M} \dot{a}_{1}-\mathbf{c}_{2} \mathbf{z}_{2}-\mathbf{z}_{1},
$$

wherec $_{2} \in \mathbb{R}^{n \times n}$ is a positive definite diagonal matrix.

Substituting equations (24) and (25) into equation (23), yields the following:

$$
\dot{V}_{2}=-\mathbf{z}_{1}^{\mathrm{T}} \mathbf{c}_{1} \mathbf{z}_{1}-\mathbf{z}_{2}^{\mathrm{T}} \mathbf{c}_{2} \mathbf{z}_{2}+\mathbf{z}_{2}^{\mathrm{T}} \mathbf{z}_{3}+\mathbf{z}_{2}^{\mathrm{T}}\left(\mathbf{d}_{1}-\widehat{\mathbf{d}}_{1}\right) .
$$

Consider that the estimation error of the mismatched disturbances $\mathbf{d}_{1}$ denoted by $\widetilde{\mathbf{d}}_{1}$ is defined as $\widetilde{\mathbf{d}}_{1}=\mathbf{d}_{1}-\widehat{\mathbf{d}}_{1}$. Now, the following Lyapunov candidate function is considered:

$$
V_{3}=V_{2}+\frac{1}{2} \tilde{d}_{1}^{T} \tilde{d}_{1}
$$

According to (26)-(27), the time derivative of $V_{3}$ becomes as follows:

$$
\dot{V}_{3} \leq-\mathbf{z}_{1}^{T} \mathbf{c}_{1} \mathbf{z}_{1}-\mathbf{z}_{2}^{T} \mathbf{c}_{2} \mathbf{z}_{2}+\mathbf{z}_{2}^{T} \mathbf{z}_{3}+\mathbf{z}_{2}^{T} \tilde{\mathbf{d}}_{1}-\tilde{\mathbf{d}}_{1}^{T} p_{2} \tilde{\mathbf{d}}_{1} .
$$

According to the generic inequality $a b \leq \varepsilon_{1} a^{2}+\left(1 / 4 \varepsilon_{1}\right) b^{2}\left(\varepsilon_{1}>0\right) \quad[18]$. Thus, we have the following:

$$
\begin{aligned}
\dot{V}_{3} & \leq-\mathbf{z}_{1}^{T} \mathbf{c}_{1} \mathbf{z}_{1}-\mathbf{z}_{2}^{T} \mathbf{c}_{2} \mathbf{z}_{2}+\mathbf{z}_{2}^{T} \mathbf{z}_{3}+\left(\mathbf{z}_{2}^{T} \varepsilon_{1} \mathbf{z}_{2}+\widetilde{\mathbf{d}}_{1}^{T} \frac{1}{4 \varepsilon_{1}} \tilde{\mathbf{d}}_{1}\right)-\tilde{\mathbf{d}}_{1}^{T} p_{2} \widetilde{\mathbf{d}}_{1} \\
& \leq-\mathbf{z}_{1}^{T} \mathbf{c}_{1} \mathbf{z}_{1}-\mathbf{z}_{2}^{T} K_{1} \mathbf{z}_{2}+\mathbf{z}_{2}^{T} \mathbf{z}_{3}-\widetilde{\mathbf{d}}_{1}^{T} K_{2} \widetilde{\mathbf{d}}_{1}
\end{aligned}
$$

where

$$
\begin{aligned}
& K_{1}=\mathbf{c}_{2}-\varepsilon_{1}, \\
& K_{2}=p_{2}-\frac{1}{4 \varepsilon_{1}} .
\end{aligned}
$$

If $\mathbf{z}_{3}=0, c_{2}$ and $\varepsilon_{1}$ are properly chosen such that $K_{1}>0$ and $K_{2}>0$, then $\dot{V}_{3} \leq 0$. Therefore, the control law design process should be reconsidered.

Step 3. According to equations (7) and (24), the derivative of $\mathbf{z}_{3}$ is computed as follows:

$$
\begin{aligned}
\dot{z}_{3} & =\dot{x}_{3}-\dot{a}_{2} \\
& =-\mathbf{R} \mathbf{x}_{3}-\mathbf{K}_{b} \mathbf{x}_{2}+\widehat{\mathbf{L}}^{-1} \mathbf{u}+\mathbf{d}_{2}-\dot{a}_{2} .
\end{aligned}
$$

Define the sliding surface $\mathbf{s}$ as follows:

$$
\mathbf{s}=k \mathbf{z}_{2}+\mathbf{z}_{3},
$$

TABLE 1: The mechanism parameters for simulation.

\begin{tabular}{lc}
\hline Parameter & Value \\
\hline$m_{p}$ & $25 \mathrm{~kg}$ \\
$m_{l 1}$ & $10 \mathrm{~kg}$ \\
$m_{l 2}$ & $10 \mathrm{~kg}$ \\
$m_{l 3}$ & $10 \mathrm{~kg}$ \\
$m_{l 4}$ & $25 \mathrm{~kg}$ \\
$m_{1}$ & $5 \mathrm{~kg}$ \\
$m_{2}$ & $5 \mathrm{~kg}$ \\
$m_{a}$ & $1.5 \mathrm{~kg}$ \\
$m_{b}$ & $1.5 \mathrm{~kg}$ \\
$a$ & $0.58 \mathrm{~m}$ \\
$b$ & $0.23 \mathrm{~m}$ \\
$c$ & $0.2 \mathrm{~m}$ \\
$r_{l 3}$ & $0.03 \mathrm{~m}$ \\
$r_{1}$ & $0.06 \mathrm{~m}$ \\
$r_{2}$ & $0.03 \mathrm{~m}$ \\
$L_{1}$ & $0.495 \mathrm{~m}$ \\
$L_{5}$ & $0.6 \mathrm{~m}$ \\
$\theta$ & $90^{\circ}$ \\
\hline
\end{tabular}

TABLE 2: The motor parameters for simulation.

\begin{tabular}{lcc}
\hline Parameters & Slider motors & $\begin{array}{c}\text { Active wheel } \\
\text { motor }\end{array}$ \\
\hline Torque constant $K_{T}$ & $0.562 \mathrm{~N} \cdot \mathrm{m} / \mathrm{A}$ & $0.959 \mathrm{~N} \cdot \mathrm{m} / \mathrm{A}$ \\
Inductance $L$ & $2.87 \mathrm{mH}$ & $3.506 \mathrm{mH}$ \\
Resistance $R$ & $1.638 \Omega$ & $0.655 \Omega$ \\
Antielectric torque constant & 0.0937 & 0.16 \\
$K_{E}$ & $200 \pi$ & 20 \\
Motor gear ratio $n$ &
\end{tabular}

where $k>0$. By differentiating from both sides of (32), the time derivative of $\mathbf{s}$ is obtained as follows:

$$
\begin{aligned}
\dot{s} & =k \dot{z}_{2}+\dot{z}_{3} \\
& =k \dot{z}_{2}-\mathbf{R} \mathbf{x}_{3}-\mathbf{K}_{b} \mathbf{x}_{2}+\widehat{\mathbf{L}}^{-1} \mathbf{u}+\mathbf{d}_{2}-\dot{a}_{2} .
\end{aligned}
$$

Define a Lyapunov candidate function as follows:

$$
V_{4}=V_{3}+\frac{1}{2} \mathbf{s}^{T} \mathbf{s}
$$

Differentiating from both sides of (34), yields the following:

$$
\begin{aligned}
\dot{V}_{4}= & \dot{V}_{3}+\mathbf{s}^{T} \dot{s} \\
= & -\mathbf{z}_{1}^{T} \mathbf{c}_{1} \mathbf{z}_{1}-\mathbf{z}_{2}^{T} K_{1} \mathbf{z}_{2}+\mathbf{z}_{2}^{T} \mathbf{z}_{3}-\widetilde{\mathbf{d}}_{1}^{T} K_{2} \widetilde{\mathbf{d}}_{1}+\mathbf{s}^{T} \\
& \cdot\left(k \dot{z}_{2}-\mathbf{R} \mathbf{x}_{3}-\mathbf{K}_{b} \mathbf{x}_{2}+\widehat{\mathbf{L}}^{-1} \mathbf{u}+\mathbf{d}_{2}-\dot{\alpha}_{2}\right) .
\end{aligned}
$$

Now, the following NDO based backstepping SMC could be designed:

$$
\begin{aligned}
\mathbf{u} & =\mathbf{u}_{1}+\mathbf{u}_{2}, \\
\mathbf{u}_{1} & =\widehat{\mathbf{L}}\left(-k \dot{z}_{2}+\mathbf{R} \mathbf{x}_{3}+\mathbf{K}_{b} \mathbf{x}_{2}-\widehat{\mathbf{d}}_{2}+\dot{\alpha}_{2}-\mathbf{z}_{2}\right), \\
\mathbf{u}_{2} & =-\widehat{\mathbf{L}} h \mathbf{s}-\widehat{\mathbf{L}} \delta \operatorname{sgn}(s),
\end{aligned}
$$



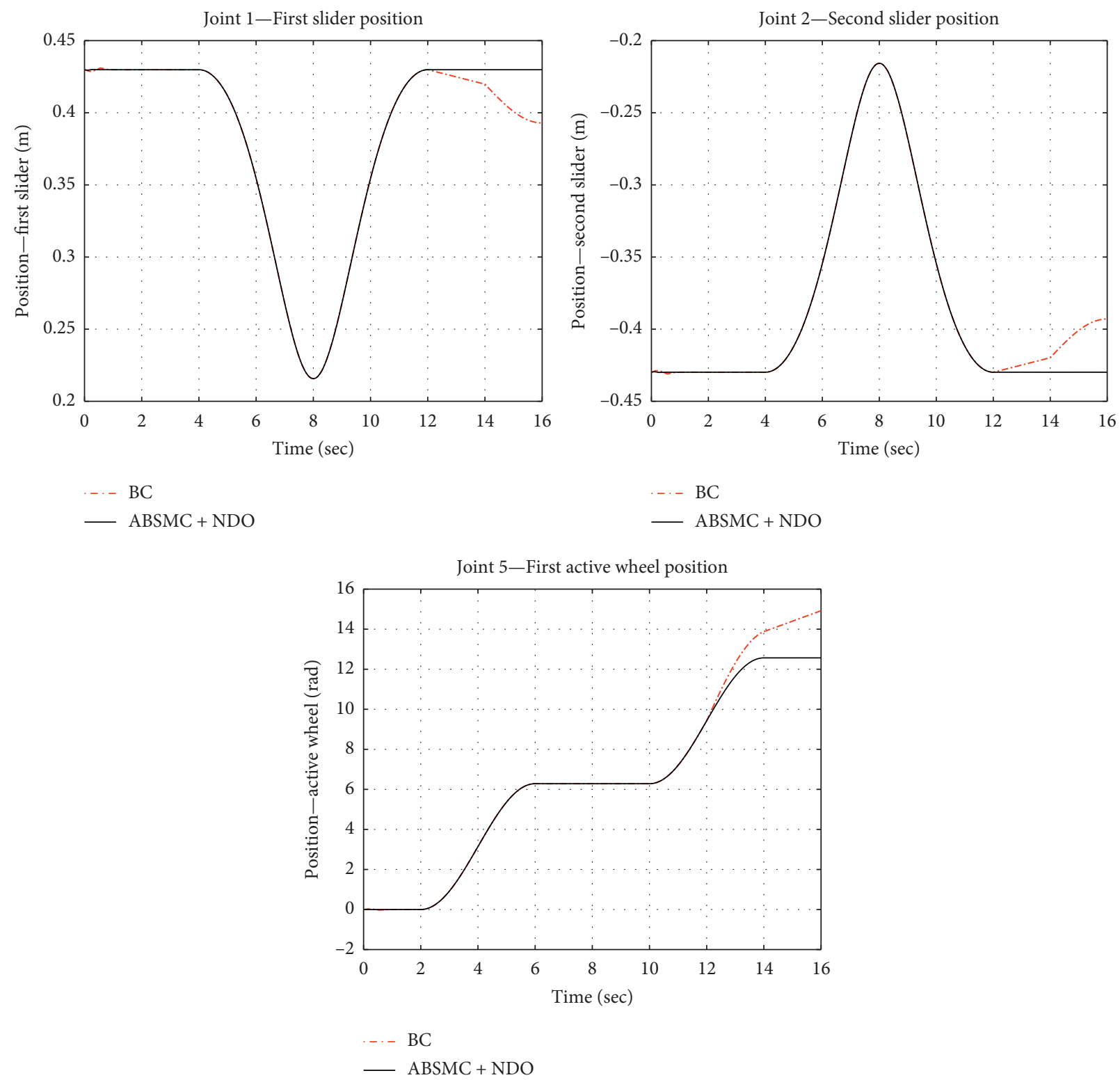

FIGURE 3: Each joint position tracking error.

where $\delta$ is a positive constant. Assume that the upper bound of $\mathbf{d}_{2}-\widehat{\mathbf{d}}_{2}$ is known and satisfies $\left\|\mathbf{d}_{2}-\widehat{\mathbf{d}}_{2}\right\|_{1} \leq \delta \leq \infty$.

Since obtaining the upper bound of $\mathbf{d}_{2}-\widehat{\mathbf{d}}_{2}$ is difficult, an appropriate adaptation mechanism is required for its estimation. This adaptive law is designed as follows:

$$
\dot{\widehat{\delta}}=\lambda\|\mathbf{s}\|_{1},
$$

where $\widehat{\delta}$ is the estimation of $\delta$, and $\lambda$ is the adaptation rate and $\lambda>0$. Define the upper bound error as $\widetilde{\delta}=\delta-\widehat{\delta}$.

According to the adaptation mechanism (38), the following hybrid control law could be obtained:

$\mathbf{u}=\widehat{L}\left(-k \dot{z}_{2}+\mathbf{R} \mathbf{x}_{3}+\mathbf{K}_{b} \mathbf{x}_{2}-\widehat{\mathbf{d}}_{2}+\dot{\alpha}_{2}-\mathbf{z}_{2}\right)-\widehat{\mathbf{L}} h \mathbf{s}-\widehat{\mathbf{L}} \widehat{\delta} \operatorname{sgn}(s)$.
Theorem 1. Consider the hybrid conveying mechanism (7), if the control law (38) is employed, the tracking error can tend to zero.

Proof. Consider a Lyapunov candidate function as follows:

$$
V_{5}=V_{4}+\frac{1}{2 \lambda} \widetilde{\delta}^{T} \widetilde{\delta}+\frac{1}{2} \widetilde{\mathbf{d}}_{2}^{T} \widetilde{\mathbf{d}}_{2} .
$$

By differentiating from both sides of (40), we have the following:

$$
\dot{V}_{5}=\dot{V}_{4}+\frac{1}{\lambda} \widetilde{\delta}^{T} \dot{\tilde{\delta}}+\widetilde{\mathbf{d}}_{2}^{T} \dot{\tilde{\mathbf{d}}}_{2} .
$$

Inserting equations (35) and (38) into equation (40) gives the following: 

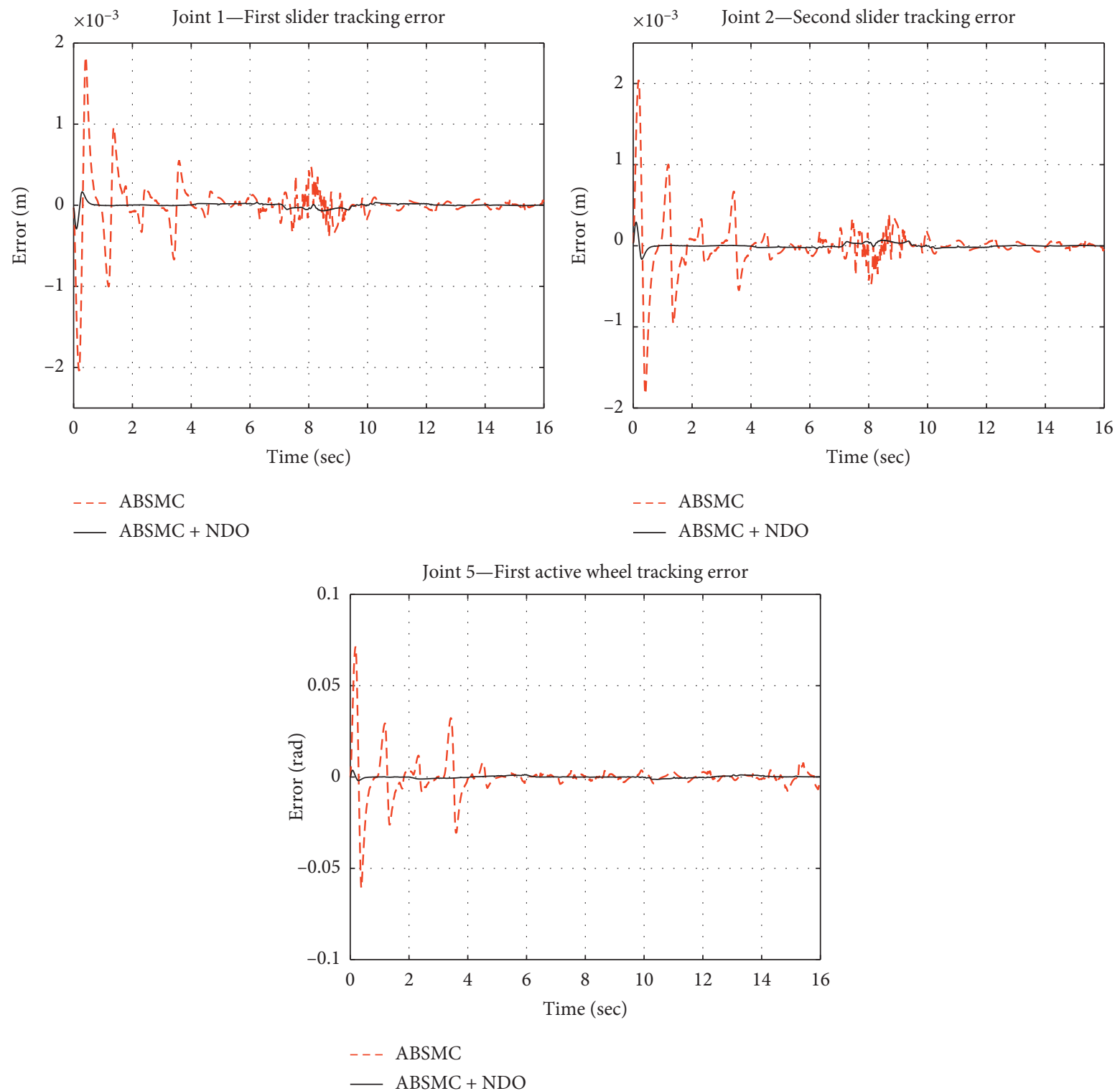

FIGURE 4: Each joint position tracking error.

$$
\begin{aligned}
\dot{V}_{5} & \leq-\mathbf{z}_{1}^{T} \mathbf{c}_{1} \mathbf{z}_{1}-\mathbf{z}_{2}^{T} K_{1} \mathbf{z}_{2}-\widetilde{\mathbf{d}}_{1}^{T} K_{2} \widetilde{d}_{1}+\mathbf{z}_{2}^{T} \mathbf{z}_{3}-\mathbf{s}^{T} \mathbf{z}_{2}-\mathbf{s}^{T} h \mathbf{s}+\mathbf{s}^{T} \widetilde{\mathbf{d}}_{2}-\mathbf{s}^{\mathrm{T}} \widehat{\delta} \operatorname{sgn}(s)-\frac{\tilde{\delta} \hat{\delta}}{\lambda}-\widetilde{\mathbf{d}}_{2}^{T} p_{3} \widetilde{\mathbf{d}}_{2} \\
& \leq-\mathbf{z}_{1}^{T} \mathbf{c}_{1} \mathbf{z}_{1}-\mathbf{z}_{2}^{T}\left(K_{1}+k\right) \mathbf{z}_{2}-\mathbf{s}^{T} h \mathbf{s}+\delta\|\mathbf{s}\|_{1}-\widehat{\delta}\|\mathbf{s}\|_{1}-\tilde{\delta}\|\mathbf{s}\|_{1}-\widetilde{\mathbf{d}}_{2}^{T} p_{3} \widetilde{\mathbf{d}}_{2} \\
& =-\mathbf{z}_{1}^{T} \mathbf{c}_{1} \mathbf{z}_{1}-\mathbf{z}_{2}^{T}\left(K_{1}+k\right) \mathbf{z}_{2}-\mathbf{s}^{T} h \mathbf{s}+(\delta-\widehat{\delta}-\tilde{\delta})\|(s)\|_{1}-\widetilde{\mathbf{d}}_{2}^{T} p_{3} \widetilde{\mathbf{d}}_{2} \\
& =-\mathbf{z}_{1}^{T} \mathbf{c}_{1} \mathbf{z}_{1}-\mathbf{z}_{2}^{T}\left(K_{1}+k\right) \mathbf{z}_{2}-\mathbf{s}^{T} h \mathbf{s}-\widetilde{\mathbf{d}}_{2}^{T} p_{3} \widetilde{\mathbf{d}}_{2} .
\end{aligned}
$$

Since $\mathrm{c}_{1}, K_{1}, k, h$, and $p_{3}$ are positive constants, $\dot{V}_{5} \leq 0$ and $\dot{V}_{5}$ is seminegative. According to equations (39), $V_{5}$ has a lower bound and $V_{5} \geq 0$. Taking the time derivative of $\dot{V}_{5}$, yields the following:

$$
\ddot{V}_{5}=-2 \mathbf{z}_{1}^{T} \mathbf{c}_{1} \dot{z}_{1}-2 \mathbf{z}_{2}^{T}\left(K_{1}+k\right) \dot{z}_{2}-2 \mathbf{s}^{T} h \dot{s}-2 \widetilde{\mathbf{d}}_{2}^{T} p_{3} \dot{\tilde{\mathbf{d}}}_{2} .
$$

Substituting equations (10), (14), (20), and (33) into (42), yields $\ddot{V}_{5}$ is bounded. Since $\dot{V}_{5}$ is seminegative, $\dot{V}_{5}$ is 

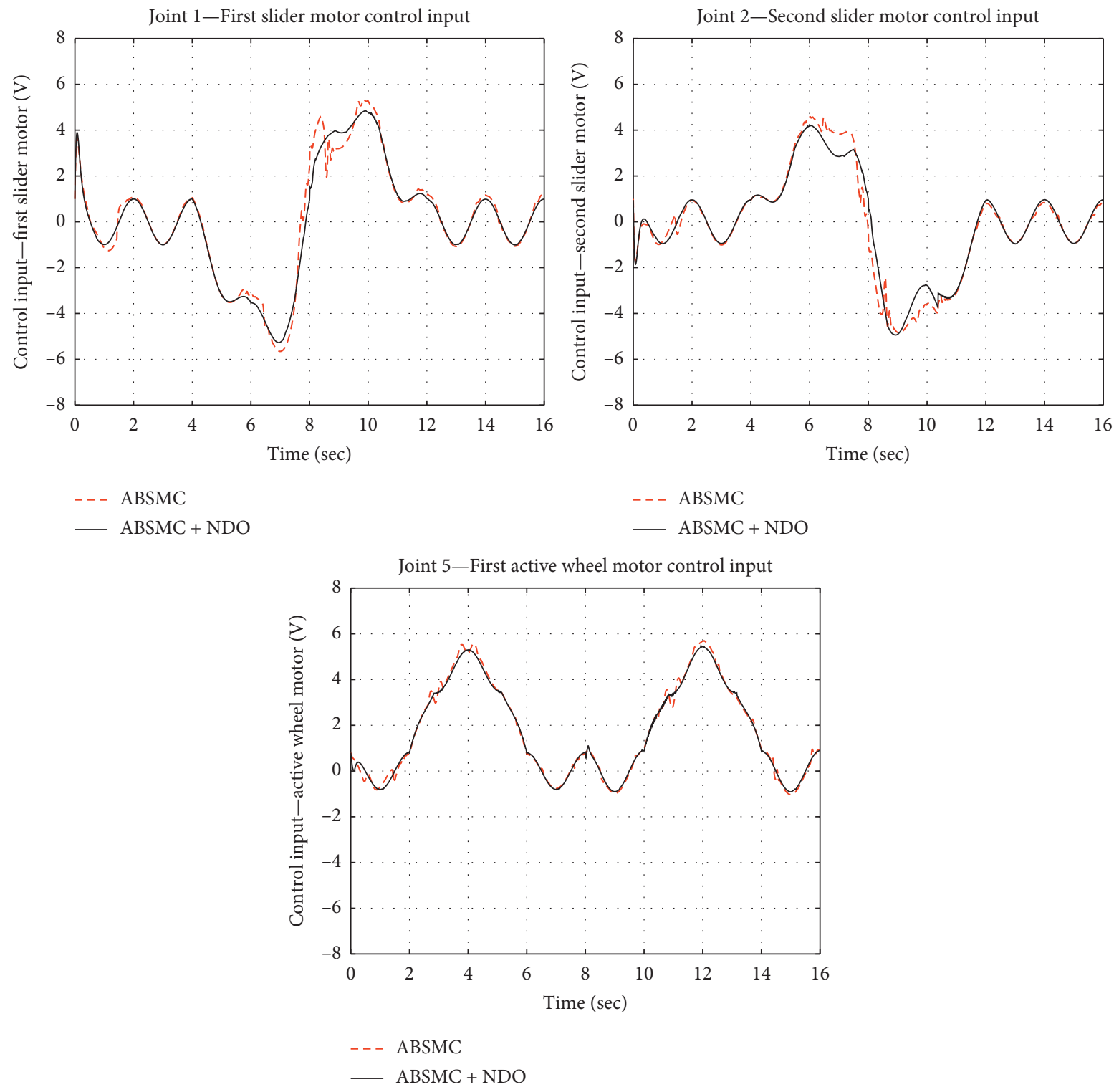

FIgURE 5: Control input of each motor.

uniformly continuous. From (41), we can get $\dot{V}_{5} \longrightarrow 0$ ast $\longrightarrow \infty$ and $\lim _{t \rightarrow \infty} \mathbf{z}_{1}=\lim _{t \rightarrow \infty} \mathbf{z}_{2}=\lim _{t \rightarrow \infty} \mathbf{z}_{3}=0$. According to the Barbalat theorem. Therefore, the tracking error can tend to zero and the closed-loop system is asymptotically stable.

\section{Numerical Simulations}

In order to evaluate the efficiency of the proposed ABSMC + NDO method, the numerical simulations are performed in this section. The parameters of the mechanism and motor for simulations are given in Tables 1 and 2, respectively. The desired trajectory is chosen the same as in [2].

In the simulations, both the suppression ability of the external disturbances and the robustness to system parameter uncertainties are considered. In practical applications, since the fluid resistance is random and large during the movement of the white body in the electrophoresis liquid, the control performance of the conveying mechanism may be degraded. Consider that the system parameters are deviated $10 \%$ from their nominal values. The external disturbances such as the fluid resistance, friction, and voltage disturbance are defined as follows:

$$
\begin{aligned}
& \mathbf{d}_{1 \mathrm{ex}}=\mathbf{d}_{\text {friction }}+\left(\mathbf{J}^{T}\right)^{+} \mathbf{d}_{\text {fluid }}, \\
& \mathbf{d}_{2 \mathrm{ex}}=2 \sin \left(\pi t+\frac{\pi}{2}\right),
\end{aligned}
$$

where $\mathbf{d}_{\text {friction }}$ is the friction and $\mathbf{d}_{\text {fluid }}$ is the fluid resistance. The friction is defined as follows:

$$
\mathbf{d}_{\text {friction }}=\mathbf{a s g n} \dot{q}+\mathbf{b} \dot{q}
$$



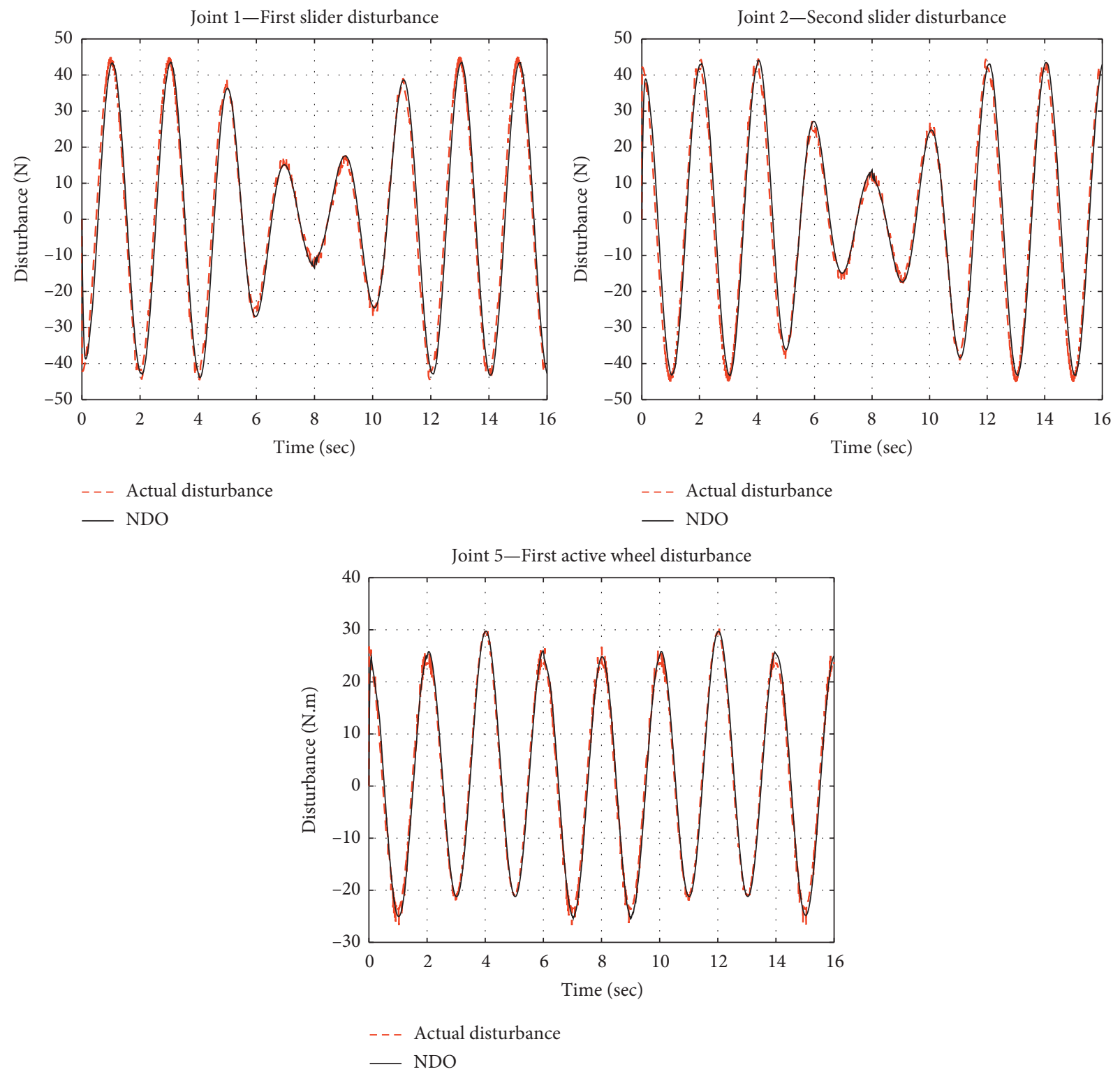

Figure 6: Estimated disturbances for each joint.

where $\mathbf{a}=\operatorname{diag}(2.0,2.0,2.0,2.0,2.0,2.0)$ and $\mathbf{b}=\operatorname{diag}(2.0$, $2.0,2.0,2.0,2.0,2.0$ ).

The fluid resistance is defined as follows:

$$
\mathbf{d}_{\text {fluid }}=\left[\begin{array}{lll}
100 \sin \left(\pi t+\frac{\pi}{2}\right) & 100 \sin \left(\pi t+\frac{\pi}{2}\right)
\end{array}\right]^{T} .
$$

To illustrate the effectiveness of the constructed hybrid control scheme (ABSMC + NDO), the backstepping control (BC) and adaptive backstepping sliding mode control (ABSMC) are utilized for comparison analysis. For better comparison and analysis, the parameters of each controller are adjusted according to their optimal control performance. The controller gains of each control scheme are given as follows:
$\mathrm{BC}$

$$
\begin{aligned}
& \mathbf{c}_{1}=\operatorname{diag}(10,10,10,10,8,8), \\
& \mathbf{c}_{2}=\operatorname{diag}(10,10,10,10,8,8), \\
& \mathbf{c}_{3}=\operatorname{diag}(15,15,15,15,10,10) .
\end{aligned}
$$

ABSMC

$$
\begin{aligned}
\lambda & =5, \\
\mathbf{c}_{1} & =\operatorname{diag}(7,7,7,7,4,4), \\
\mathbf{c}_{2} & =\operatorname{diag}(7,7,7,7,4,4), \\
\mathbf{k} & =\operatorname{diag}(6,6,6,6,4,4), \\
\mathbf{h} & =\operatorname{diag}(5,5,5,5,3,3) .
\end{aligned}
$$



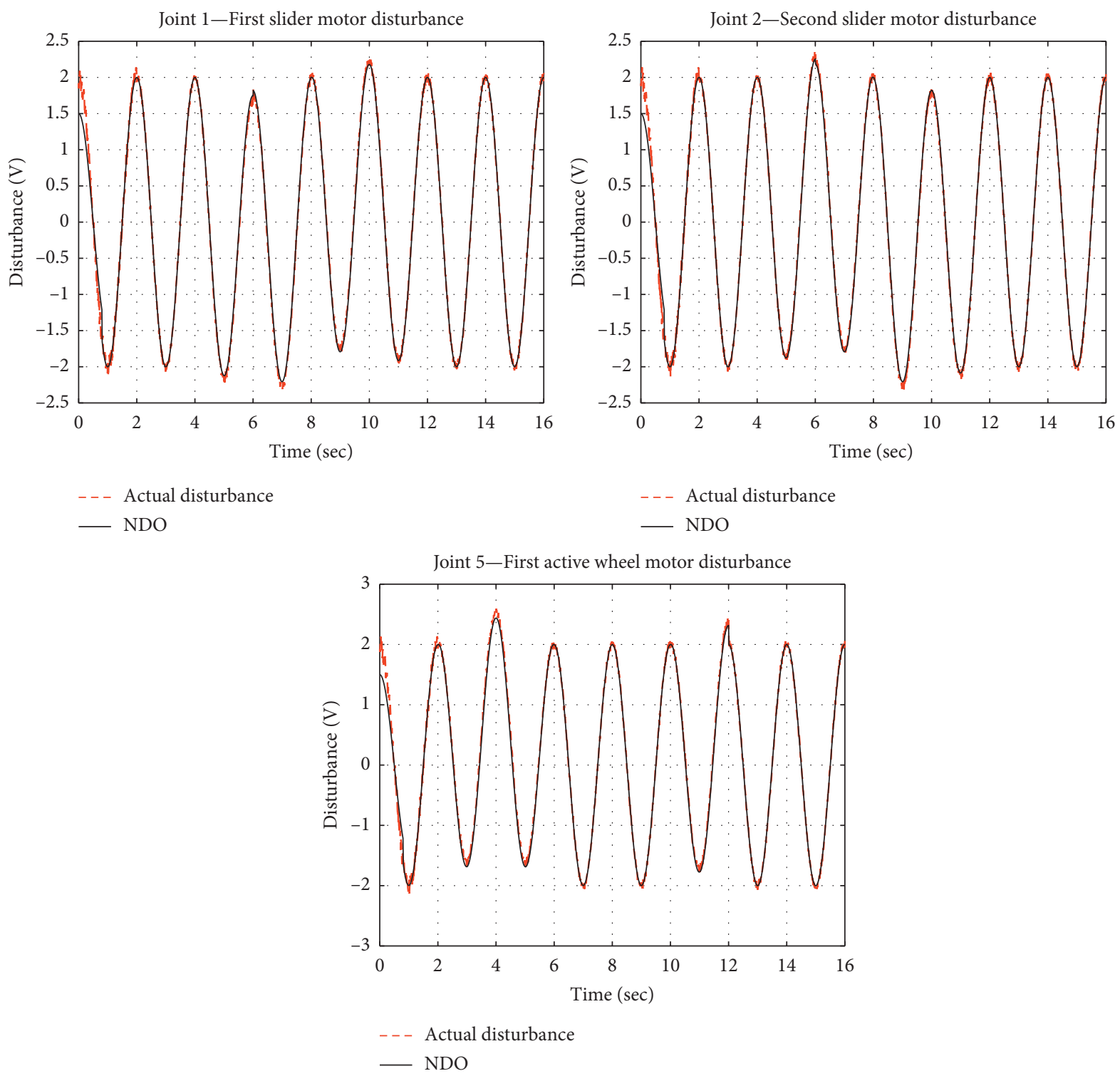

FIGURE 7: Estimated disturbances for each motor.

TABLE 3: RMSEs for each joint.

\begin{tabular}{|c|c|c|c|}
\hline \multicolumn{2}{|c|}{ Control scheme } & ABSMC & $\mathrm{ABSMC}+\mathrm{NDO}$ \\
\hline \multirow{3}{*}{ RMSE } & First slider & $27.1 \times 10^{-5}$ & $3.4 \times 10^{-5}$ \\
\hline & Second slider & $27.1 \times 10^{-5}$ & $3.4 \times 10^{-5}$ \\
\hline & Active wheel & $7.8 \times 10^{-3}$ & $6.1 \times 10^{-4}$ \\
\hline
\end{tabular}

$$
\begin{aligned}
\mathrm{ABSMC} & +\mathrm{NDO} \\
\lambda & =5, \\
\mathbf{c}_{1} & =\operatorname{diag}(4.8,4.8,4.8,4.8,3,3), \\
\mathbf{c}_{2} & =\operatorname{diag}(4.8,4.8,4.8,4.8,3,3), \\
\mathbf{k} & =\operatorname{diag}(4,4,4,4,3,3), \\
\mathbf{h} & =\operatorname{diag}(3,3,3,3,2,2), \\
p_{2} & =\operatorname{diag}(600,600,600,600,400,400), \\
p_{3} & =\operatorname{diag}(150,150,150,150,120,120)
\end{aligned}
$$

The following two cases are considered in the simulation.

4.1. Case 1. Compared with the backstepping control, the disturbances of the system are added to the system at $t=12 \mathrm{~s}$. Due to the symmetry of the HCM, just the simulation results of the first slider, the second slider, and the first active wheel are given in this paper. Figure 3 is the position tracking curve of the HCM. It can be obtained from Figure 3 that the slider trajectories are the same during the first $12 \mathrm{~s}$ when the system has no disturbances, and the backstepping control cannot track the 


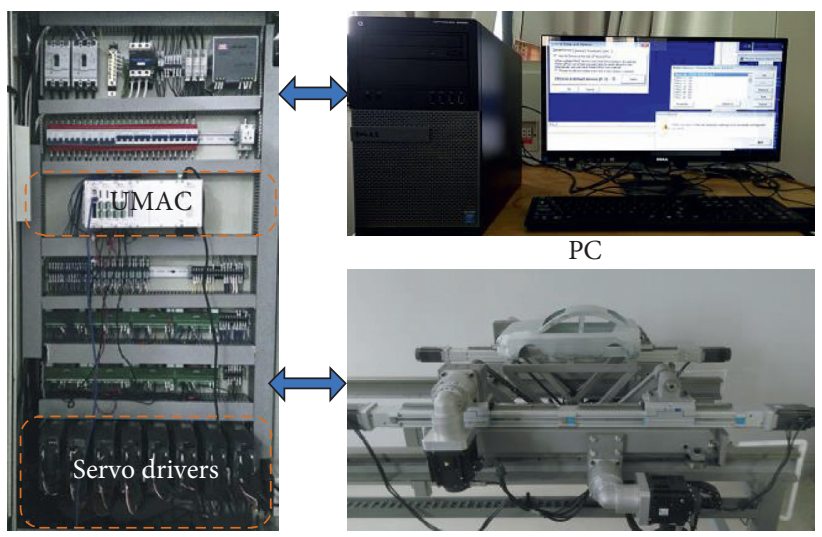

Electrical cabinet

Hybrid conveying manipulator

FIgURE 8: The hybrid conveying mechanism prototype.
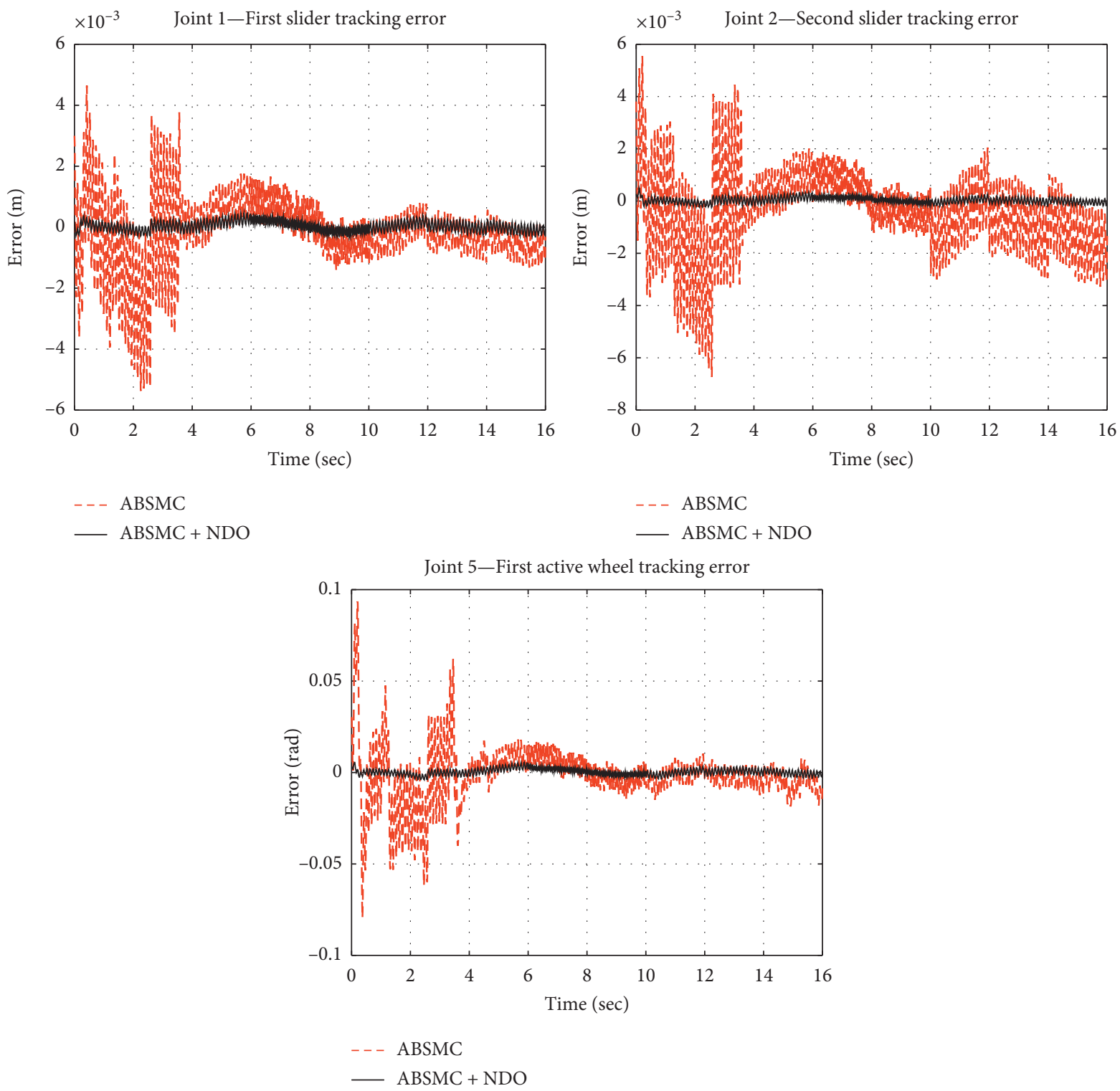

Figure 9: The experimental results of each joint. 
given trajectory when $t \geq 12 \mathrm{~s}$, which illustrates the backstepping control has poor robustness and the proposed ABSMC + NDO has excellent disturbance rejection performance.

4.2. Case 2. Compared with the adaptive backstepping sliding mode control, the disturbances of the system are added to the system from the beginning. Figures 4-7 shows the simulation results for two control schemes. Figure 4 depicts the trajectory tracking error of the HCM for the given trajectory with two distinct control strategies. As could be seen in Figure 4, although the desired trajectory could be tracked by using both of these control schemes, small tracking error could be observed with the proposed control scheme. The control signals for the two control approaches are presented in Figure 5. It could be concluded from Figure 5 that the control signal of the presented control scheme is substantially smooth and the chattering phenomenon could be attenuated through the proposed control scheme. The root mean square error (RMSE) values for two distinct control strategies are presented in Table 3. The estimated disturbances are shown in Figures 6 and 7. According to two figures, the disturbance estimation error is small, and the disturbances could be effectively estimated via the NDO with appropriate precision. As shown in Figures 4-7 and Table 3, the proposed control scheme reduces the trajectory tracking error, requires small control torque, alleviates the chattering problem, and has superior mismatched disturbance rejection performance when the system introduces the NDO accurately estimates and compensates for the mismatched disturbances.

\section{Prototype Experiments}

To further verify the effectiveness of the proposed control strategy, we use the HCM prototype shown as Figure 8 for experimental verification. The control system of the prototype includes a host computer (PC), a slave computer (UMAC), eight servo drives and motors. The PC and UMAC are connected through the Ethernet cable. The main task of the PC is to initialize the system, implement the control algorithm, manage the data, and monitor the operating status. On the other hand, UMAC is employed for servo motor control and acquisition of the encoder position and the limit switch signals. The UMAC consists of the main CPU board (PMAC2 OPT-5C0), two four-axis motion servo control boards (ACC-24E2A OPT-1A), an I/ $\mathrm{O}$ interface board (ACC-65E), and a power supply board (ACC-E1).

The reference trajectory employed in the simulation is also utilized in the experiment. The experiment results are shown in Figure 9. A small gain is utilized in the experiment to attenuate the measurement noise. It could be observed from Figure 8 that greater trajectory tracking errors are obtained in the experiment compared with the corresponding ones obtained in simulations. However, the proposed control scheme has a small trajectory tracking error and high tracking precision.

\section{Conclusion}

In this paper, a hybrid control scheme is constructed for trajectory tracking control of the electrically driven hybrid conveying mechanism with mismatched disturbances and parameter uncertainties. The main contribution of this paper is that the proposed controller not only compensates for mismatched disturbances but also effectively reduces sliding mode chattering based on NDO. The novel hybrid control scheme combines the NDO, backstepping, and SMC to reject mismatched disturbances. The Lyapunov stability theory is used to ensure the closed-loop system stability and tracking on the given trajectory. Simulation and experimental results are provided to demonstrate the validity of the proposed control scheme. Designing the adaptive NDO gain and the optimal disturbance rejection strategy could be considered as the future work.

\section{Data Availability}

The data used to support the findings of this study are available from the corresponding author upon request.

\section{Conflicts of Interest}

The authors declare that there are no conflicts of interest regarding the publication of this paper.

\section{Acknowledgments}

This research was funded in part by National Natural Science Foundation of China, grant nos. 51375210 and 61503162, and Blue Project of Jiangsu Province.

\section{References}

[1] Y. Cao, G. Gao, and X. Wu, "Synchronization control based on novel error for a hybrid mechanism," Proceedings of the Institution of Mechanical Engineers, Part I: Journal of Systems and Control Engineering, vol. 231, no. 9, pp. 717-728, 2017.

[2] W. Yuan and G. Gao, "Disturbance observer-based adaptive integral sliding mode control for the hybrid automobile electrocoating conveying mechanism," International Journal of Advanced Robotic Systems, vol. 16, no. 3, 2019.

[3] M. A. Khosravi and H. D. Taghirad, "Robust PID control of fully-constrained cable driven parallel robots," Mechatronics, vol. 24, no. 2, pp. 87-97, 2014.

[4] W. Shang and S. Cong, "Nonlinear computed torque control for a high-speed planar parallel manipulator," Mechatronics, vol. 19, no. 6, pp. 987-992, 2009.

[5] Z. Chu, J. Cui, and F. Sun, "Fuzzy adaptive disturbanceobserver-based robust tracking control of electrically driven free-floating space manipulator," IEEE Systems Journal, vol. 8, no. 2, pp. 343-352, 2014.

[6] M. Furqan, M. Suhaib, and N. Ahmad, "Studies on Stewart platform manipulator: a review," Journal of Mechanical Science and Technology, vol. 31, no. 9, pp. 4459-4470, 2017.

[7] A. Salimi Lafmejani, M. Tale Masouleh, and A. Kalhor, "Trajectory tracking control of a pneumatically actuated 6DOF Gough-Stewart parallel robot using backstepping-sliding mode controller and geometry-based quasi forward 
kinematic method," Robotics and Computer-Integrated Manufacturing, vol. 54, pp. 96-114, 2018.

[8] W. Yuan and G. Gao, "Sliding mode control of the automobile electrocoating conveying mechanism with a nonlinear disturbance observer," Advances in Mechanical Engineering, vol. 10, 2018.

[9] V. Utkin, J. Guldner, and J. Shi, Sliding Mode Control in Electro-Mechanical Systems, pp. 147-150, CRC Press, London, UK, 2009.

[10] J. Yang, A. Zolotas, W.-H. Chen, K. Michail, and S. Li, "Robust control of nonlinear MAGLEV suspension system with mismatched uncertainties via DOBC approach," ISA Transactions, vol. 50, no. 3, pp. 389-396, 2011.

[11] X. Wang, X. Chen, and L. Wen, "The LQR baseline with adaptive augmentation rejection of unmatched input disturbance," International Journal of Control, Automation and Systems, vol. 15, no. 3, pp. 1302-1313, 2017.

[12] D. Ginoya, P. D. Shendge, and S. B. Phadke, "Sliding mode control for mismatched uncertain systems using an extended disturbance observer," IEEE Transactions on Industrial Electronics, vol. 61, no. 4, pp. 1983-1992, 2014.

[13] J. Yang, S. Li, and X. Yu, "Sliding-mode control for systems with mismatched uncertainties via a disturbance observer," IEEE Transactions on Industrial Electronics, vol. 60, no. 1, pp. 160-169, 2013.

[14] M. Rubagotti, A. Estrada, F. Castanos, A. Ferrara, and L. Fridman, "Integral sliding mode control for nonlinear systems with matched and unmatched perturbations," IEEE Transactions on Automatic Control, vol. 56, no. 11, pp. 2699-2704, 2011.

[15] F. Castanos and L. Fridman, "Analysis and design of integral sliding manifolds for systems with unmatched perturbations," IEEE Transactions on Automatic Control, vol. 51, no. 5, pp. 853-858, 2006.

[16] J. Zhang and F. Zhu, "Output control of MIMO system with unmatched disturbance based on high-order unknown input observer," International Journal of Control, Automation and Systems, vol. 15, no. 2, pp. 575-584, 2017.

[17] T. D. Thien, D. X. Ba, and K. K. Ahn, "Adaptive backstepping sliding mode control for equilibrium position tracking of an electrohydraulic elastic manipulator," IEEE Transactions on Industrial Electronics, vol. 65, no. 5, pp. 3860-3869, 2019.

[18] Y.-H. Lan and Z. Lei-Zhou, "Backstepping control with disturbance observer for permanent magnet synchronous motor," Journal of Control Science and Engineering, vol. 2018, Article ID 4938389, 8 pages, 2018.

[19] L. Ma, K. Schilling, and C. Schmid, “Adaptive backstepping sliding-mode control with application to a flexible-joint manipulator," IFAC Proceedings Volumes, vol. 39, no. 16, pp. 55-60, 2006.

[20] N. Chen, F. Song, G. Li, X. Sun, and C. Ai, "An adaptive sliding mode backstepping control for the mobile manipulator with nonholonomic constraints," Communications in Nonlinear Science and Numerical Simulation, vol. 18, no. 10, pp. 2885-2899, 2013.

[21] K. Bai, X. Gong, S. Chen, Y. Wang, and Z. Liu, "Sliding mode nonlinear disturbance observer-based adaptive back-stepping control of a humanoid robotic dual manipulator," Robotica, vol. 36, no. 11, pp. 1728-1742, 2018.

[22] W. H. Chen, D. J. Balance, and P. J. Gawthrop, "A nonlinear disturbance observer for robotic manipulators," IEEE Transactions on Industrial Electronics, vol. 47, pp. 932-938, 2000.
[23] S. Li, J. Yang, and W. H. Chen, Disturbance Observer-Based Control Methods and Applications, CRC Press, London, UK, 2014.

[24] A. Mohammadi, M. Tavakoli, H. J. Marquez, and F. Hashemzadeh, "Nonlinear disturbance observer design for robotic manipulators," Control Engineering Practice, vol. 21, no. 3, pp. 253-267, 2013.

[25] Y. Singh and M. Santhakumar, "Inverse dynamics and robust sliding mode control of a planar parallel (2-PRP and 1-PPR) robot augmented with a nonlinear disturbance observer," Mechanism and Machine Theory, vol. 92, pp. 29-50, 2015.

[26] S. Liu, Y. Liu, and N. Wang, "Nonlinear disturbance observerbased backstepping finite-time sliding mode tracking control of underwater vehicles with system uncertainties and external disturbances," Nonlinear Dynamics, vol. 88, no. 1, pp. 465476, 2016

[27] Q. Ai, C. Zhu, J. Zuo et al., "Disturbance-estimated adaptive backstepping sliding mode control of a pneumatic musclesdriven ankle rehabilitation robot," Sensors, vol. 18, no. 2, p. 66, 2017.

[28] J. Zhang, X. Liu, Y. Xia, Z. Zuo, and Y. Wang, "Disturbance observer-based integral sliding-mode control for systems with mismatched disturbances," IEEE Transactions on Industrial Electronics, vol. 63, no. 11, pp. 7040-7048, 2016.

[29] C. Peng, J. Fang, and X. Xu, "Mismatched disturbance rejection control for voltage-controlled active magnetic bearing via state-space disturbance observer," IEEE Transactions on Power Electronics, vol. 30, no. 5, pp. 2753-2762, 2015.

[30] H. Sun, S. Li, L. Guo, and J. Yang, "Non-linear disturbance observer-based back-stepping control for airbreathing hypersonic vehicles with mismatched disturbances," IET Control Theory \& Applications, vol. 8, no. 17, pp. 1852-1865, 2014.

[31] M. Khamar and M. Edrisi, "Designing a backstepping sliding mode controller for an assistant human knee exoskeleton based on nonlinear disturbance observer," Mechatronics, vol. 54, pp. 121-132, 2018. 